\title{
Cellular mechanisms of motor control in the vibrissal system
}

\author{
Michael Brecht • Valery Grinevich • Tae-Eun Jin • \\ Troy Margrie $\cdot$ Pavel Osten
}

Received: 14 April 2006 / Accepted: 2 May 2006 / Published online: 31 May 2006

(C) Springer-Verlag 2006

\begin{abstract}
In this article we discuss the experimental advantages that the vibrissal motor system offers for analysis of motor control and the specializations of this system related to the unique characteristics of whisker movements. Whisker movements are often rhythmic, fast, and bilateral. Movements of individual whiskers have simple characteristics, whereas, movements of the entire vibrissae array are complex and sophisticated. In the last few years, powerful methods for high precision tracking of whisker movements have become available. The whisker musculature is arranged to permit forward movements of individual whiskers and consists - depending on the species - mainly or exclusively of fast contracting, fast fatigable muscle fibers. Whisker motor neurons are located in the lateral facial nucleus and their cellular properties might contribute to the rhythmicity of whisking. Numerous structures provide input to the lateral facial nucleus, the
\end{abstract}

M. Brecht $(\square)$

Department of Neuroscience, Erasmus MC,

Postbus 17388, 3000 DR Rotterdam, The Netherlands

e-mail: m.brecht@erasmusmc.nl

V. Grinevich · P. Osten

Department of Molecular Neurobiology,

Max Planck Institute for Medical Research,

Jahnstrasse 29, 69120 Heidelberg, Germany

T.-E. Jin

Department of Physiology, Center for Molecular Medicine,

Samsung Biomedical Research Institute,

Sungkyunkwan University School of Medicine,

300 Chunchun-dong Jangan-ku, Suwon 440-746, South Korea

T. Margrie

The Wolfson Institute for Biomedical Research,

Department of Physiology, University College London,

Gower Street, London WC1E 6BT, UK most mysterious and important one being the putative central pattern generator (CPG). Although recent studies identified candidate structures for the $\mathrm{CPG}$, the precise identity and the functional organization of this structure remains uncertain. The vibrissa motor cortex (VMC) is the largest motor representation in the rodent brain, and recent work has clarified its localization, subdivisions, cytoarchitectonics, and connectivity. Single-cell stimulation experiments in VMC allow determining the cellular basis of cortical motor control with unprecedented precision. The functional significance of whisker movements remains to be determined.

Keywords Vibrissa motor cortex - Whisker tracking . Whole-cell recording · Single-cell stimulation - Whisker . Facial nucleus

\section{Introduction}

In this article, we discuss the potential that the vibrissal motor system holds as a model system for motor control. The facial vibrissae function as important tactile sensors in most mammalian species [1-3], and as in other sensory systems, active positioning of sensors is a prominent feature of the vibrissal system. Whereas the sensory side of the vibrissal system, in particular, the vibrissa to barrel cortex pathway, is an established model for cortical sensory pattern formation and information processing $[4,5]$, the vibrissal motor system has received less attention. As a result of the brief research history, major issues in vibrissal motor control are yet to be resolved. We will, therefore, not present a synthesis on how the multiple components of the vibrissal motor system interact; neither will we discuss sensorimotor interactions in this system. There are, howev- 

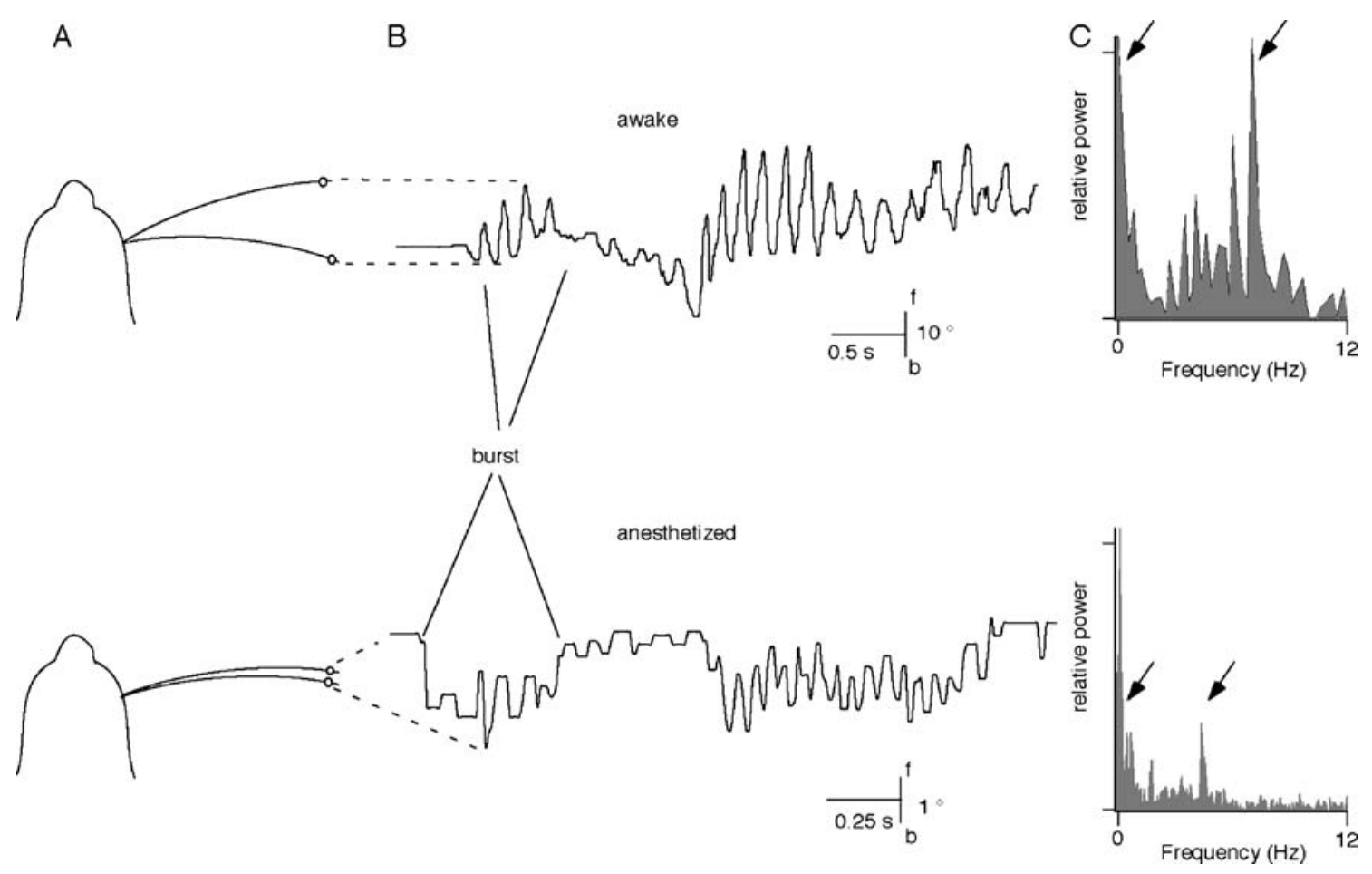

Fig. 1 Whisker movements in awake and anesthetized animals. a Schematic representation of a dorsal view of a rat's snout. Top: two whisker positions observed in an awake animal are illustrated. Only one whisker, which carries a reflex foil label, is drawn. Bottom: two whisker positions observed in an animal anaesthetised with ketamine/ acepromazine. b Top: movement trace of whisking in an awake animal; bottom: whisking movements in an anaesthetised animal. Note the different scaling of the two traces. c Power spectra of the movements shown in $\mathbf{b}$ er, excellent reviews available on these issues [6, 7]. Instead, we focus on selected aspects of the vibrissal motor system, including the whisking behavior, whisker musculature, and the vibrissa motor cortex (VMC). Whisker movements are fascinating, as they are some of the fastest -yet quite sophisticated - movements performed by mammals. In our review, we discuss two broad topics. First, we point out the experimental advantages that the vibrissal motor system offers for the analysis of motor control. Second, we discuss specializations of the vibrissal motor system that we recently identified and that might be relevant to the unique characteristics of whisker movements.

\section{Whisker movements and whisking behavior}

Whisking behavior Most mammals can move their facial whiskers. However, the degree to which whiskers are mobile differs among species. Some rodents, for example, rats, hamsters, and mice, display a highly specialized pattern of whisker movements referred to as "whisking" [8-11]. Whisking behavior consists of strictly rhythmic back and forth movements and occurs at high repetition rates, up to $25 \mathrm{~Hz}$. It can involve large amplitude whisker movements $\left(>100^{\circ}\right)$ and occurs mainly in the plane of the whisker row. Figure 1 (top row) shows a whisking episode recorded in an awake rat. Whisking is a high-speed behavior with repetition rates that exceed those of most other behaviors displayed by mammals.

Special features of vibrissal movement Our article-as most of the experimental work done so far-deals with the motor control of whisker movements in rats and mice. The vibrissa motor system of these rodents has a variety of unique features: (1) as already noted, movements tend to be rhythmic; (2) whisker movements can be extremely fast; (3) many of the whisker movements are bilaterally symmetric; (4) the movements of individual whiskers have relatively simple movement characteristics; (5) the movements of the entire vibrissae array are complex and sophisticated.

Temporal organization of whisker movements The temporal characteristics of whisker movements have been studied in a number of rodents. In whisking species, vibrissa sweeps follow in close succession with typical frequencies of around $5-15 \mathrm{~Hz}$ in rats (Fig. 1 top row $[8,11]$ ) and $15-$ $25 \mathrm{~Hz}$ in mice $[10,12]$. It is also becoming clear that 
Table 1 Methods for whisker movements

\begin{tabular}{|c|c|c|c|c|c|}
\hline Technique & $\begin{array}{l}\text { Temporal } \\
\text { resolution }\end{array}$ & $\begin{array}{l}\text { Spatial } \\
\text { resolution }\end{array}$ & Major advantage & Major drawback & $\begin{array}{l}\text { Application } \\
\text { Cost }\end{array}$ \\
\hline Mystacial EMG & $<1 \mathrm{~ms}$ & - & $\begin{array}{l}\text { Muscle specific } \\
\text { information }\end{array}$ & $\begin{array}{l}\text { No direct whisker position } \\
\text { read out }\end{array}$ & $\begin{array}{c}\text { Invasive } \\
\text { cheap }\end{array}$ \\
\hline Optoelectronic monitoring & $<1 \mathrm{~ms}$ & $<0.1^{\circ}$ & Fast & Whisker labeling & Cheap \\
\hline Video-based tracking & $\geq 16.7 \mathrm{~ms}$ & Up to $0.01^{\circ}$ & Easy & Whisker labeling or cutting & Cheap \\
\hline $\begin{array}{l}\text { High-speed camera-based } \\
\text { tracking }\end{array}$ & $<1 \mathrm{~ms}$ & Up to $0.01^{\circ}$ & Fast & Whisker labeling or cutting & Costly \\
\hline
\end{tabular}

whisking species are capable of multiple modes and frequencies of whisking. Thus, in rats, Berg and Kleinfeld [13] described a special form of "foveal whisking" with a frequency of 15-25 Hz. Similarly, hamsters seem to engage in distinct forms of whisking with different frequencies [9]. Whisker movements are not only rhythmic but also occur in bursts during which the amplitude of whisker movements waxes and wanes (Fig. 1). Within bursts, the whisking frequencies are highly constant, i.e., whisking bursts are spectrally pure [13].

Simple and complex spatial movement characteristics Whiskers can be individually identified and follow a stereotyped topography (five rostrocaudally running rows labeled A-E from dorsal to ventral, and within rows, whiskers are numbered incrementally from posterior to anterior). The movements of individual whiskers have relatively simple characteristics, which can be approximated as lever-like movements around the whisker origin. This description is mathematically very simple (a one-dimensional back and forth movement) but still captures much of the variance of these movements $[8,11,14,15]$. In contrast to the simple synchronous movements of whiskers during free whisking, rats execute complex whisker movements of the whisker array when they actively palpate an object, i.e., when their whiskers are in an object contact (Brecht, unpublished). Thus, several studies have described relatively independent, divergent whisker movements in behaving animals $[16,17]$.

Escape from sleep-/anesthesia-induced paralysis Much like eye movements, whisker movements can escape from sleep- or anesthesia-induced paralysis [18, 19]. As shown in Fig. 1, for spontaneously occurring whisking epochs, whisking amplitudes are larger, and whisking frequencies are higher in awake rats than in anesthetized animals. However, in both cases, movements are rhythmic and occur in bursts. The fact that at least some movement characteristics can be studied in lightly anesthetized animals offers enormous experimental advantages [20].
Functional significance of whisker movements Only very few studies have addressed this central question in research on the vibrissal motor system. Although transection of the facial nerve is easily performed and robustly abolishes whisker movements, few researchers have performed such manipulation in combination with behavioral testing. Surprisingly, one of such tests led to a negative result, $i$. e., there was no effect of facial nerve transsection on a whisker task, where an aperture width had to be estimated [21]. Clearly, further work must be directed to the question, "What are whisker movements are good for?"

\section{Quantifying whisker movements}

The quantification of whisker movements poses considerable experimental difficulties. These difficulties arise from three sources: (1) whiskers move at high velocities, requiring short exposure times and a fast recording technique to image; (2) whiskers are thin with little optical contrast; (3) among the 30 whiskers of the array, it is difficult to identify and follow the movements of single whiskers. In fact, in the first study on whisking by Welker [8], whisking was observed mainly in blind rats, as seeing animals would not perform whisking under the high light levels required for the high speed photography used to document the whisker movements. Fortunately, in the last few years, a variety of powerful methods to document whisker movements have become available (Table 1). In fact, a highly precise tracking of movements is now a strength of research on the vibrissal motor system.

Mystacial EMG recordings Electro-myographic recordings from the mystacial pad were pioneered by Carvell and colleagues [22]. By simultaneously recording mystacial EMG and monitoring whisker position by a videocamera, these authors showed that EMG activity allows estimating whisker position. EMG recordings have an excellent temporal resolution and such recordings can be selectively 

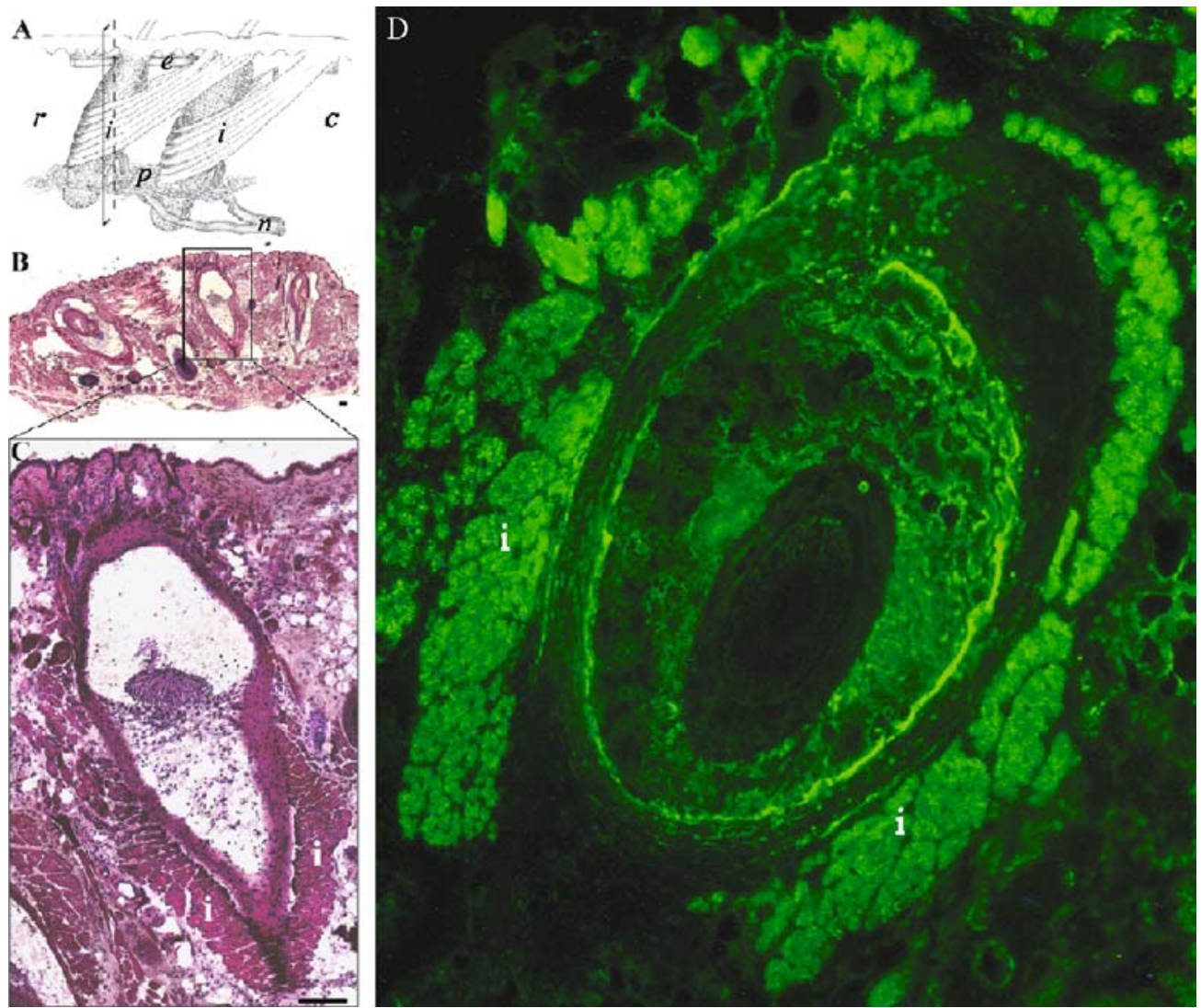

Fig. 2 Mystacial pad musculature. a Schematic overview of vibrissa follicles (after Dörfl 1982 [25]). $r$ Rostral, $c$ caudal, $n$ motor nerve, $p$ plate, $i$ intrinsic whisker muscle, $e$ extrinsic whisker muscle; rectangle marks longitudinal section across mystacial pad as shown in (b) and (c). b Mystacial pad stained with hematoxylin/eosin. c Magnified view from $\mathbf{b}$ as outlined by a rectangle. $i$ Intrinsic whisker muscle. d Mouse intrinsic whisker muscle fibers surrounding a whisker follicle stained with NOQ7.5.4.D (GFP-labeled) antibodies to detect myosin heavy chain 2 (MyHC2). Intrinsic whisker muscle fibers are arranged around the whisker follicle (the oval structure in the center of the image) and are without exception positive for $\mathrm{MyHC2}$. Thus, mouse intrinsic whisker muscle fibers are all of the fast contracting muscle fiber type. Within the follicle, unspecific labeling can be seen. $i$ Intrinsic whisker muscle. Scale bars: $100 \mu \mathrm{m}$. a-c Modified from [12] targeted to extrinsic or intrinsic muscle groups [13]. This way, EMG recordings may allow insights that cannot be obtained with other tracking techniques. The drawback of EMG recordings is that whisker position is only estimated and that single-whisker tracking is currently not possible.

Optoelectronic monitoring An array of light-sensitive elements was used by Zeigler and colleagues for a highspeed online monitoring of whisker position [23]. This method allows single-whisker tracking. The method has an excellent temporal resolution and a good spatial resolution, but under many circumstances, requires labeling of whiskers.

Video-based systems Video-based analysis of whisker movements was first performed by Carvell and Simons [11]. They removed all but one row of whiskers and were then able to track the movements of multiple individual whiskers. The temporal resolution of video recordings is limited (Table 1), but it is possible to boost video sampling rates with stroboscopic techniques [12]. If one uses small reflective whisker labels, multiple individual whiskers can be tracked without whisker removal. Small labels do not seem to alter whisker movements [12], but it is difficult to exclude all label effects. A drawback from most early video-based systems was that they required extensive offline processing to extract whisker positions. However, an automatized online tracking solutions have recently become available [20]. Video-based systems are cheap, have an excellent spatial resolution and allow the tracking of multiple individual whiskers.

High-speed camera-based systems These systems have, in principle, similar properties as video-based systems but can have a much better temporal resolution. Thus, whisker movement can be tracked with an unprecedented accuracy [24]. High-speed cameras vary in price, but this is nevertheless, the most costly tracking technique. 

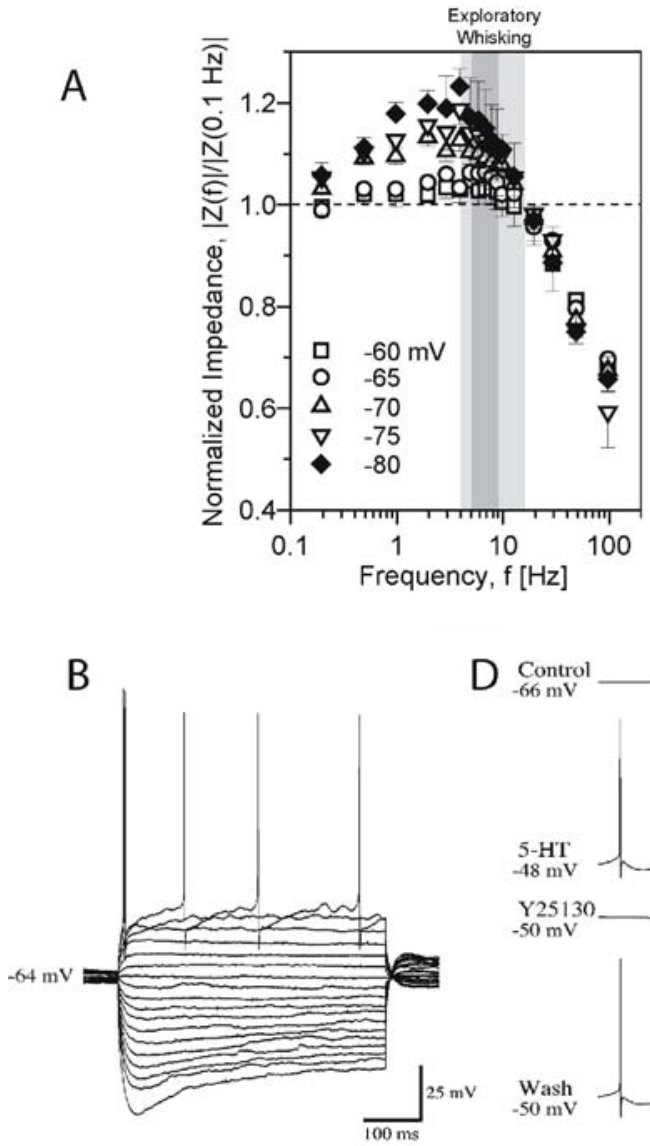

D Control

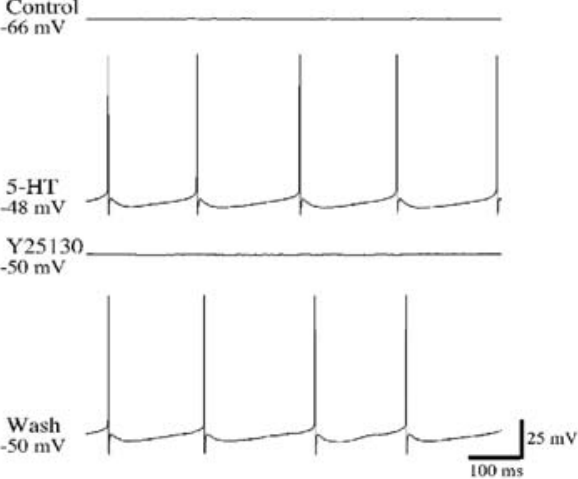

C

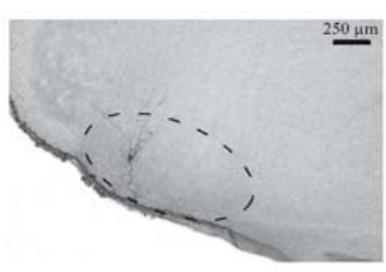

$\mathrm{E}$

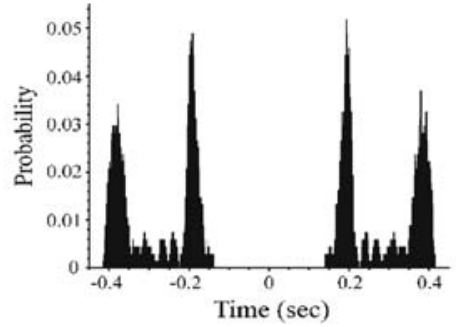

Note the absence of plateau potentials and the appearance of hyperpolarization-activated current (Ih) only at deeply hyperpolarized potentials, indicating that wFMNs are not intrinsically bursting. Exogenous application of serotonin $(30 \mu \mathrm{M})$ evokes repetitive firing at regular intervals (c), which is reversibly abolished by the selective 5-HT3 receptor antagonist Y25130 (10 $\mu \mathrm{M}$; [C]). d Photomicrograph of a biocytin-labeled wFMN in the lateral facial nucleus. e The autocorrelogram of interspike intervals of serotonin-evoked firing in the wFMNs shown in $(\mathbf{C})$ shows that the cell is firing rhythmically at $5.2 \mathrm{~Hz}$. Modified from [34]

\section{Whisker musculature}

Whiskers are moved by an extensive and elaborate set of muscles. The structural and physiological characteristics of this musculature form the basis for the fast and individual movement of whiskers.
Intrinsic and extrinsic whisker muscles Dörfl was the first to delineate the structural basis of whisker movements [25]. Two classes of muscles generate movements of whiskers. One class (shown schematically in Fig. 2a), the intrinsic whisker muscles (also referred to as vibrissal capsular muscle), has their points of attachment completely within 
the mystacial pad and forms a sling around each follicle [25]. ( $i$ in Fig. 2a and c). Intrinsic whisker muscles constitute the majority of the whisker pad musculature, and their contraction causes protraction (forward movement) of the vibrissae $[13,22]$. A second class of muscles, the extrinsic muscles ( $e$ in Fig. 2a.), anchors external to the mystacial pad, and their contraction results in retraction (backward movement) of the vibrissae [13, 26].

Muscle fiber composition of whisker muscles Muscle fiber types differ in the stability and fatigue resistance of their contractions and in the maximum velocity of contraction. Type 1 fibers, so called slow fibers, generally have high levels of aerobic oxidative enzymes and show stability and fatigue resistance. Type 2 fibers, so called fast fibers, can be subdivided into four types. Type $2 \mathrm{~A}$ fibers have oxidative enzymes, and are thus, fatigue resistant. Type $2 \mathrm{~B}$ and $2 \mathrm{D}$ fibers have high levels of anaerobic glycolytic enzymes, providing a rapid oxygen-independent source of ATP; however, this pathway rapidly depletes glycogen stores and fatigue develops after brief usage [27]. To better characterize whisker musculature, we recently determined muscle fiber types of intrinsic whisker muscles [12]. In the hematoxylin/eosin-stained sections shown in Fig. 2b,c, the intrinsic whisker muscle can be recognized by their dark $\mathrm{red} / \mathrm{violet}$ color. As shown in Fig. 2d, almost all of the mouse intrinsic whisker muscle fibers show immunoreactivity for the myosin heavy chain 2 (MyHC2), i.e., they are of the fast contracting muscle fiber type. Further analysis based on RT-PCR of MyHC transcripts, ATPase staining of MyHC isoforms, and antibody staining showed that whisking musculature of rats and mice is unique in its large fraction of type $2 \mathrm{~B}$ fibers. Type $2 \mathrm{~B}$ fibers provide the highest twitch velocity $[28,29]$ but are at the same time highly susceptible to fatigue. These observations suggest that the need for high contraction speed may have been a driving force in the evolution of whisker muscles.

\section{Subcortical control of whisker movements}

Much like research on the sensory side of the vibrissal system, investigators of the motor system have focused much more on the central cortical representations than on the peripheral levels of the motor pathway. As a result, our knowledge of the subcortical mechanisms of vibrissal motor control is rather sketchy and will be discussed here only briefly.

Lateral facial nucleus There is a consensus that the cholinergic motor neurons of the lateral facial nucleus are the sole output station innervating and controlling whisker muscles. Facial nucleus cells are large neurons, with dendrites extending beyond the facial nucleus into the reticular formation [30]. The number of facial nucleus vibrissa motor neurons is on the order of 1,000 to 2,000 cells, i.e., 50 to 100 per whisker [31]. As one might expect, stimulation of motor neurons at the level of the facial nerve can evoke whisker movements [32]. As shown in Fig. 3a, their resonance properties [33] and the cellular effects of serotonergic inputs (Fig. 3b-e) [34] may predispose facial nucleus motor neurons to rhythmic discharges. Facial nucleus neurons receive direct sensory input from trigeminal brainstem neurons [35], as well as widespread inputs from other motor centers, some of which will be discussed below [36].

The central pattern generator Perhaps the least understood structure in the vibrissal motor control is the putative central pattern generator $(\mathrm{CPG})$ for whisker movements. Behavioral and deafferentation data suggest that whisking may be generated in a feedback-independent manner, i.e., by a relatively autonomous CPG [37]. The idea that a brainstem CPG controls whisking received first support by the work of Welker [8], which showed that decorticated animals display whisking behaviors. The persistence of whisking after removal of the entire cerebral cortex primed researchers to look for a subcortical whisking CPG. Thus, anatomical [36] and physiological/pharmacological [34] studies delineated brain stem circuits involved in the control of whisking. Brainstem neurons, including serotonergic cells that provide inputs to the whisking motor neurons in the facial nucleus, may form the brainstem pattern generator for whisking. Deviating from this idea, a recent study proposed an unorthodox suggestion that the inferior olive may act as the CPG for whisking [38]. Taken together, it is clear that although a CPG is playing a central role in the generation of a whisking behavior, our understanding of this structure remains preliminary.

Superior colliculus and other subcortical vibrissal motor structures Sensorimotor signaling occurs at all levels from connections between brainstem sensory and motor neurons to high-level sensorimotor interactions between cortical areas and cortico-cerebellar interactions [6]. Among the many subcortical structures contributing to vibrissal motor control, the superior colliculus plays a particularly important role. The colliculus not only receives and represents vibrissal inputs but also sends direct inputs to the facial nucleus $[6,36]$. The superior colliculus also receives massive input from the VMC [39].

\section{Organization of the vibrissa motor cortex}

The VMC is the largest motor representation in the brain of most rodents. VMC contains an estimated 1-1.5 million 
neurons [20] and takes up $\sim 45 \%$ of the entire rat primary motor cortex (M1) and about $4.5 \%$ of the total volume of the rat neo- and allocortex [40, 41]. Our review focuses on recent information about rat VMC, which became available in the last few years.

Localization and topography Rat motor cortex was first identified by surface stimulation techniques [42]. Since then, a variety of microstimulation studies [43-47] have further characterized the functional organization of rat primary motor cortex. VMC is characterized by low $(<60 \mu \mathrm{A})$ thresholds for whisker movement initiation. In many of the microstimulation studies, VMC was described as extended along the anterior-posterior axis and narrow along the anteroposterior axis. Subsequent analysis showed, however, that the appearance of VMC as a narrow medial stripe is deceiving and might be related to the use of vertical mapping penetrations in the strongly curved rat motor cortex. As illustrated in Fig. 4, the VMC representation extends into the medial bank of the rat brain (Fig. 4b, c), and the layout and proportions of the motor map compare to the somatosensory cortical map (Fig. 4a). The number of moving whiskers varies as a function of the stimulation site, and multi-whisker movements often encompass the whiskers of a row. At threshold, in $25 \%$ of stimulation sites, it is possible to evoke single- or dualwhisker movements [41]. Overall, the general topography of VMC is now agreed on (Fig. 4a,b). How well is the body topography preserved in rat motor cortical representations remains, however, a matter of debate.

Multiple subdivisions Recent work in awake animals demonstrated that VMC contains at least two functionally distinct representations [48]. In the major (anterior) part of VMC, the "retraction-face" region, stimulation with short or long microstimulation pulse trains evokes a single backward whisker movement and concomitant facial movements. In a smaller (posteromedial) part of VMC, the "rhythmic whisking" region, stimulation with long microstimulation pulse trains evokes continuous whisking. There may be further vibrissa motor cortical representations besides these two subdivisions of primary VMC. According to the data of Neafsey et al. [46], the very rostral parts of the rat cortex seem to contain an additional small motor area with more or less complete musculotopic body representation; this area may represent the rat's secondary motor cortex (M2).

Cytoarchitectonics of VMC There is an excellent relationship between cytoarchitectonics and motor maps in the rat motor cortex (Fig. 4). Contrary to earlier reports [49], VMC corresponds to the so-called agranular medial area $\left(\mathrm{AG}_{\mathrm{m}}\right)$. This conclusion is not only supported by microstimulation data $[46,50]$ but is also evident from the intracellular stimulation of identified cells [41]. The conspicuous cytoarchitectonic specializations of $\mathrm{VMC} /$ area $\mathrm{AG}_{\mathrm{m}}$ makes it one of the most distinct areas of the rat cortex. Thus, the area $A_{\mathrm{m}}$ is characterized by an expanded layer 5 (Fig. $4 \mathrm{c}$, g) and a strong myelinization (Fig. 4e). The strong myelinization and-as discussed below-also the expanded layer 5 of area $\mathrm{AG}_{\mathrm{m}}$ may represent cytoarchitectonic specializations related to the control of the high-speed whisking behavior. Finally, it is noteworthy that, so far, there is no report of an anatomical manifestation of a single vibrissa motor aggregate that would be analogous to the somatosensory barrels. Instead, it appears to be the case that vibrissa motor representations are less discrete and focally organized than their somatosensory counterparts, much like in other motor cortices [51].

Connectivity of VMC VMC is heavily synaptically connected to the somatosensory barrel cortex [52]. A recent anatomical study [53] demonstrated that S1 inputs from vibrissa rows converge in VMC. VMC, in turn, projects selectively to septal columns of barrel cortex [54]. Like other cortices, VMC projects to the thalamus and the superior colliculus. What is noteworthy about the overall pattern of VMC outputs is the strongly bilateral nature of the projections [36]. VMC also targets a variety of brainstem nuclei (Fig. 5), some of which may form the CPG for whisking [34]. Recently, we demonstrated, by lentivirus-based expression of GFP and GFP-tagged presynaptic proteins, the existence of monosynaptic VMC input to facial nucleus motoneurons (Fig. 5d-h) [55]. Such direct corticomotoneuronal input is unusual in the mammalian nervous system, where motor cortex typically innervates motor neurons indirectly, via oligosynaptic pathways. The rat VMC corticomotoneuronal pathway might be an evolutionary analogy to primate corticomotoneuronal pathways. In primates, abundant monosynaptic cortical projections onto spinal motor neurons innervating distal limb muscles emerged in parallel with the evolution of skilled digit movements [56].

Functional role of the VMC The way VMC governs whisker movements is an active area of research. Kleinfeld and colleagues accumulated evidence suggesting that VMC might be able to act as a pattern generator for whisking. Specifically, this group observed covariation of motor cortical activity and whisking [57], whisking evoked by intracortical microstimulation (ICMS) [58] and sensory tuning for whisking frequencies in motor cortex [59]. According to other models, VMC does not act as a pattern generator for whisking but merely gates the brain stem whisking generator [34]. In line with this idea, unitrecording studies in VMC [60] did not observe a one to 

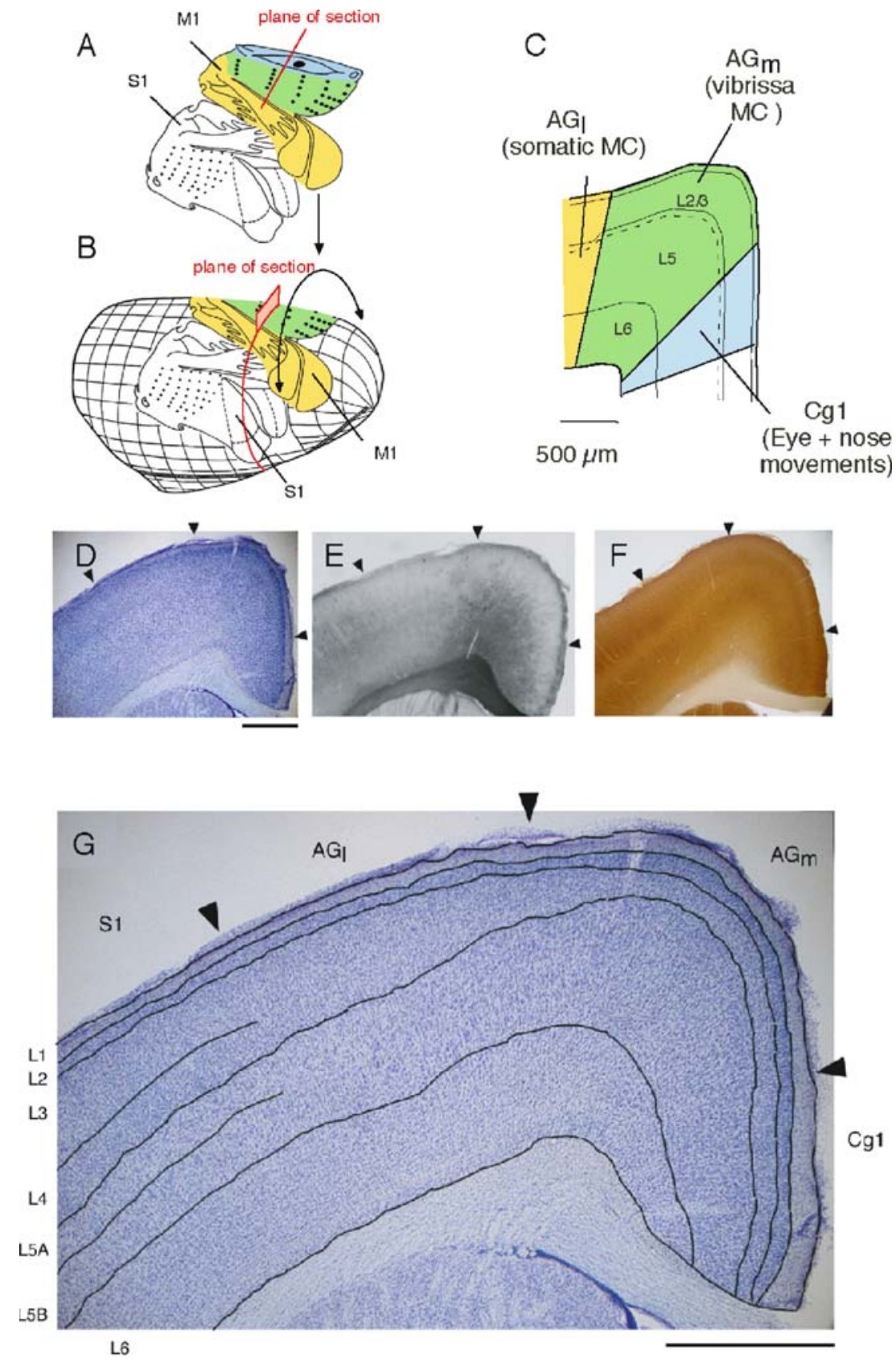

Fig. 4 Localization and cytoarchitectonics of rat vibrissa motor cortex. a Flat maps of rat primary motor (M1) and somatosensory (S1) cortices. b Dorsolateral view of a rat cortical hemisphere with superimposed M1 and S1 maps. c Coronal section through M1. Light blue, cingulate area $1(\mathrm{Cg} 1)$ (eye movement motor cortex); green, the agranular medial field $\left(\mathrm{AG}_{\mathrm{m}}\right)$ (vibrissa motor cortex) and agranular lateral field $\left(\mathrm{AG}_{1}\right)$ (somatic motor cortex). d-f Three adjacent $100-\mu \mathrm{m}$

one relationship between cortical activity and the whisking cycle. It seems also plausible, though, that both models are true and that the VMC is able to govern whisking in multiple distinct ways [61]. Lesion studies first indicated a persistence of whisking after decortication [8]. More recent studies demonstrated alterations in whisking after cortical lesions $[62,63]$, but the induced deficits were often subtle sections from rat motor cortex are shown. d Nissl stain. e Myelin stain (after McNally and Peters). A dark silver precipitate reveals myelin. f Stain for cytochrome $\mathrm{C}$ oxidase activity. Increased activity is revealed by a dark/brownish color. $\mathbf{g}$ Same as in $\mathbf{d}$ but at higher magnification and with highlighted layer boundaries. The scale bar below the lower right corner of each section equals $1 \mathrm{~mm}$. Modified from [41]

and did not immediately indicate a specific cortical function. All in all, the function of VMC is still very much unclear, in part, also because of the lack of understanding of the overall significance of whisker movements. What is required are studies that apply high precision whisker tracking and manipulation of VMC activity during behavioral paradigms, which necessitate whisker movement. 


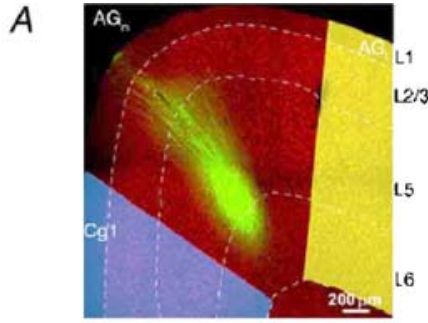

B

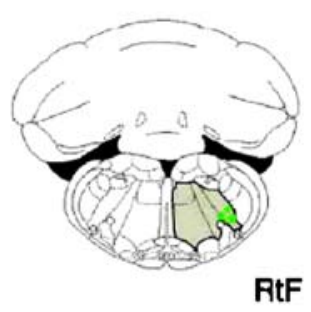

RtF

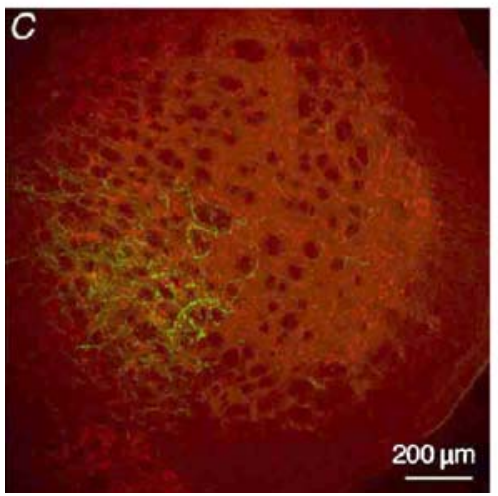

Fig. 5 Lentivirus-based GFP tracing of reticular and corticomotoneuronal VMC output. a Injection site of a GFP-expressing lentivirus in layer 5 of VMC. Confocal image of the injection area (4 weeks after lentivirus injection) shows GFP-expressing pyramidal neurons in the $\mathrm{Ag}_{\mathrm{m}}$ area (vibrissa motor cortex), mainly in the layer 5. GFP signal was enhanced with anti-GFP followed by FITC-conjugated secondary antibodies, neuronal somata were stained anti-NeuN followed by Texas Red-conjugated secondary antibodies. The neighboring $\mathrm{Cg} 1$ and $\mathrm{AG}_{1}$ regions are shown overlaid with blue and yellow, respectively. b Topography of GFP-positive fibers in the intermediate and parvocellular reticular formation (schema as in panel $\mathbf{a}$; three asterixes indicate dense labeling). c GFP-positive axons make dense plexus surround NeuN-positive neurons in the parvocellular reticular nucleus. d Topography of GFP-positive fibers (marked by a green asterix) in the lateral FN (schema adapted from [72]. e Distribution of GFP-

\section{Analysis of cortical motor control by single-cell stimulation}

In the last section of the review, we discuss single-cell stimulation experiments in the rat VMC. To this end, highprecision whisker tracking is combined with whole-cell recordings, which are relatively easy to obtain in anesthetized rodents [64]. This experimental approach allows insights that so far could not be obtained in other motor systems. positive fibers in the lateral FN. The FN area (large outline by white line) was determined based on morphological identification of facial motor neurons stained with anti-NeuN antibodies. f GFP-positive fibers in the FN at high magnification. The image is an enlargement from the area marked by a square outline in the panel e. GFP-positive fibers were found in close appositions to large NeuN positive facial motor neurons (arrows). $\mathbf{g}$ VMC axons form synapses on facial motor neurons confirmed by electron microscopy. Two GFP-positive axons (marked by asterix) are located near the soma of a motor neuron 4 weeks after multiple injection of lentivirus into VMC. GFP immunoproduct was visualized by diaminobenzidine (h). Enlarged image from panel (g) showing a GFP-positive axonal terminal with spherical vesicles in a synaptic contact with the motor neuron perikarya. Modified from [55]

The rationale of single-neuron stimulation experiments Beginning with the discovery of motor cortex by Fritsch and Hitzig [65], stimulation techniques played a key role in the analysis of motor systems. However, most present-day stimulation techniques, like transcranial magnetic stimulation, brain surface stimulation, or microstimulation, do not apply stimulation currents directly to the individual neurons to be stimulated. As a consequence, these techniques rely on large stimulation currents and the brain is activated in a 
not so well controlled fashion; with these techniques, it is also impossible to identify the exact neuronal elements that were activated. Even for microstimulation [66], which is the most focal technique of the population stimulation approaches, the estimates of how many cells are activated by a given current range over several orders of magnitude [67]. In contrast, intracellular recording techniques (sharp microelectrode or whole-cell recordings) provide a highly controlled stimulation, as the stimulated neuron can be easily identified, and spike train parameters can be accurately controlled. It is, therefore, surprising that in vivo single-cell stimulation studies are rarely applied. One reason for the absence of single-cell stimulation experiments may be the assumption that single-cell stimulation would be ineffective, given the large number of neurons in the brain. However, this is not the case [20, 68]. Surprisingly, the movements evoked by single-cell stimulation in the VMC are quite complex and of a long duration [20]. At more peripheral levels, the classic single somatosensory afferent stimulation experiments by Vallbo and colleagues $[69,70]$ have demonstrated perceptual relevance of single neuron and even single-spike activity. Similarly, single motor neuron activity is known to be able to generate considerable force [71]. Besides the experimental evidence for single-cell stimulation effects, there is a strong conceptual motivation for exploring the motor consequences of single-cell activity by stimulation experiments.

Cell identity predicts motor effects in VMC Whole-cell recordings allow post hoc identification of the stimulated neuron. It is, thus, possible to analyze the relationship between cell morphology/location and evoked movement effects. For layer 5 pyramidal cells, evoked rhythmic movements have a constant phase relative to the action potential (AP) train, indicating that single layer 5 pyramids can reset the rhythm of whisker movements. In contrast, APs evoked in layer 6 pyramids can generate bursts of rhythmic whisking, with a variable phase of movements relative to the AP train [20]. It, thus, seems that the lamination of motor cortex mirrors particular aspects of whisking behavior, namely, L5 the phase of the whisking movement, and L6, the bursting pattern of whisking. Pyramidal cell stimulation generally evoked movements of the same direction as nearby extracellular stimulation (Fig. 6a-d), suggesting that pyramidal cell activity may mediate the effects of extracellular stimulation. Different effects, however, were observed after stimulation of putative inhibitory interneurons, with fast spiking AP responses to current injection (note that the sample size of the interneuron population was quite small though). Figure $6 \mathrm{e}-\mathrm{h}$ illustrates a stimulation experiment in a fast spiking L6 interneuron, classified as a multipolar cell (Fig. 6e). At the site, where the neuron was recorded, mirostimulation evoked backward movements of several whiskers (Fig. 6h). In contrast, an intracellular current injection, which elicited a high frequency train of APs in the interneuron (55 APs at $180 \mathrm{~Hz}$ ), evoked forward movements of the same whiskers (Fig. $6 \mathrm{~b}$ right column). Injection of only ten APs at $50 \mathrm{~Hz}$ did not evoke clear-cut whisker movements. Stimulation of two other interneurons evoked similar initial movements in a direction opposite from the ICMS-evoked movement, with no movement after injections of ten APs at $50 \mathrm{~Hz}$.

Single-cell stimulation and neural codes for whisker movements An intriguing issue is how a small number of APs evoked in a single neuron can be so effective in the extensive M1 network. It seems possible that firing of a single cell may entrain a large number of target neurons. However, it is unclear how interneuron stimulation, which probably elicits inhibition of ongoing AP activity in postsynaptic neurons, can be explained by such a nonlinear amplification. The fact that interneuron activity can evoke movements - presumably by inhibiting spiking in other cells - may also indicate that the ongoing activity of motor cortical neurons is involved in the tonic control of whisker position. Overall, it appears that AP activity in the vibrissal system is read out with the outmost precision.

The variation of spike train parameters in single-cell stimulation experiments allows for the determining of how neurons encode movement characteristics. Recent work in VMC suggests that increasing AP number decreases latency to movement onset, whereas, AP frequency determines movement direction and amplitude.

\section{Concluding remarks}

Our understanding of vibrissal motor control is still quite incomplete. Following the initial description of whisker movements by Welker [8] in the last 20 years, dramatic progress was made in the quantification and analysis of these movements $[9,11,14]$. This ongoing progress is driven, at least in part, by technical advances in the quantification of whisker movements [11, 22-24]. The basic anatomy [25, 26] and physiology $[12,13,26]$ of the whisker musculature have been worked out, although detailed quantitative information is missing. Neurons in the lateral facial nucleus are the whisker motor neurons and some of their cellular properties might contribute to the rhythmicity of whisking [33, 34]. Numerous structures provide input to the lateral facial nucleus, including the mysterious putative CPG. Though recent studies identified candidate structures for the CPG $[34,36,38]$, we do not yet know, for certain, what this structure is and how it works. VMC is the most intensely 

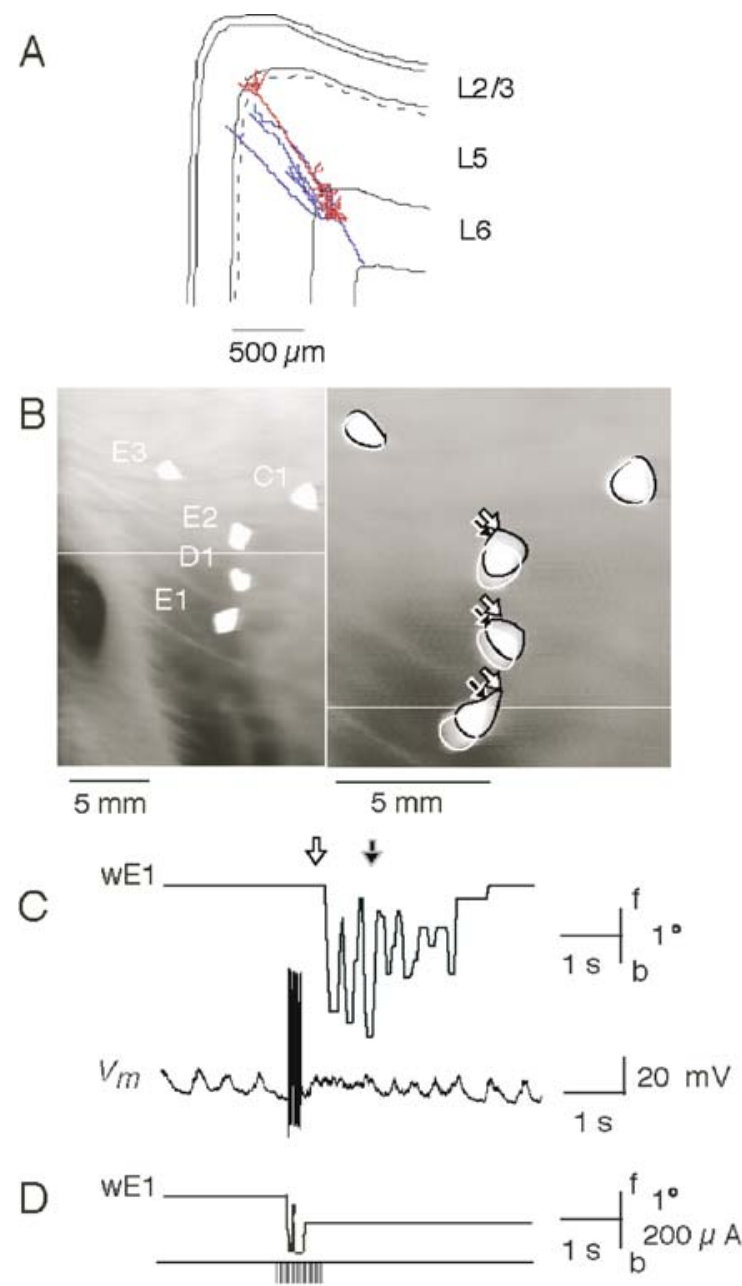

Fig. 6 Effects of pyramidal cell, interneuron, and extracellular stimulation in VMC. a Topographic position of the stimulated L6 pyramidal cell with its dendritic (red) and axonal (blue) arbor. b Left: top view of rat head and labeled whiskers; Right, enlarged superimposed images of pre- and poststimulation of whisker positions (marked by arrows). ctop trace: whisker position of whisker E1 during an intracellular stimulation trial. Evoked are $10 \mathrm{APs}$ at $50 \mathrm{~Hz}$. Lower traces: Membrane potential recordings and injection pattern of depolarizing current steps during an intracellular stimulation trial. d Top trace: whisker position of whisker E1 during an extracellular stimulation (applied close to the recorded neuron) trial. Bottom trace: extracellular stimulation current. e Topographic position of the
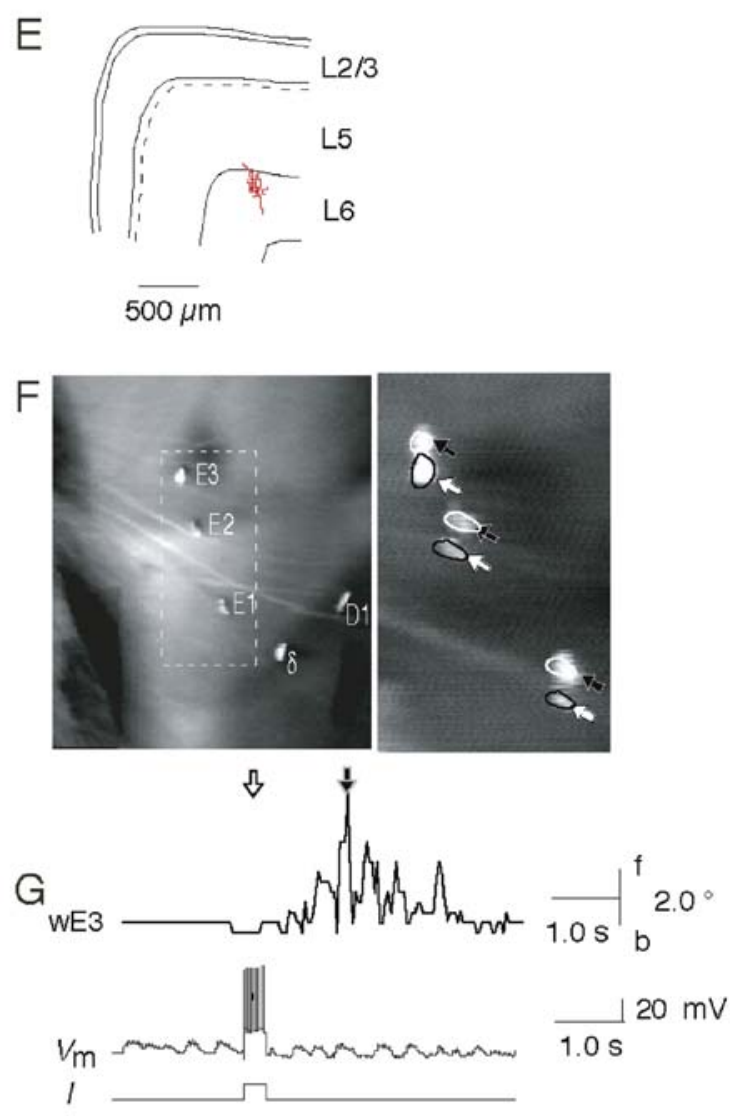

$\mathrm{HWE3}$ rNAT

III

stimulated multipolar interneuron with its dendritic (red) arbor. f Left: top view of rat head and labeled whiskers. Right, enlarged superimposed images of pre- and poststimulation of whisker positions (marked by arrows). g Top trace: whisker position of whisker E1 during an intracellular stimulation trial. Lower traces: membrane potential recordings and injection pattern of depolarizing current steps during an intracellular stimulation trial. In this trial such current injection evoked $55 \mathrm{APs}$ at around $180 \mathrm{~Hz}$. h Top trace: whisker position of whisker E1 during an extracellular stimulation (applied close to the recorded neuron) trial. Bottom trace: Extracellular stimulation current. (a-d) Modified from [55]

Experimental advantages of vibrissal motor system for the analysis of motor control A number of features of the vibrissal motor system make it an attractive system to analyze movement generation:

(1) The movement characteristics of individual whiskers are simple.

(2) Whisker movements can be tracked with high precision. on the function of whisker movements, and the functic significance of whisker movements remains to be delineated. investigated vibrissal motor structure. Recent work clarified the localization [41, 46], subdivisions [48], cytoarchitectonics [41], and connectivity of VMC [52-55]. Single-cel stimulation experiments in VMC allow for determining the cellular basis of cortical motor control with unprecedented precision [20]. There has been only little behavioral analysis 
(3) Rhythmic whisking can be quantitatively characterized by spectral analysis techniques.

(4) Load plays only a subordinate role.

(5) Whiskers and probably also whisker musculature can be easily and individually manipulated.

(6) Miniature whisker movements escape from anesthesiaor sleep-induced paralysis.

(7) Vibrissal motor representations are very large in the rodent brain.

(8) Many, though not all, parts of the vibrissal motor system have been at least partly characterized.

(9) Rodents are superb experimental preparations (cost, accessibility, genetics).

The large number of novel publications on vibrissal motor research shows that the potential of this system is more and more widely recognized.

Specializations of the vibrissal motor system There is a number of recurring themes in vibrissal research. The vibrissal system is clearly specialized for high-speed motor control. Whisker movements are extremely fast, the whisker musculature consists almost entirely of fast-contracting, fastfatigable fibers, and the VMC is strongly myelinated. Rhythmicity is another such theme. Whisking is strikingly rhythmic, facial nucleus motorneurons might be predisposed to rhythmic discharges, VMC subregions and layers differ in the rhythmicity of stimulation evoked movements, and the rhythmicity of VMC activity is debated. Another recurring topic is the segregated control of individual or at least subsets of whiskers. Fractionated whisker movements are evident at the behavioral level, the intrinsic whisker musculature is organized to allow such movements, single-whisker movements can be evoked by stimulation from various whisker motor structures, and - evolutionary - the direct corticomotoneuronal vibrissal pathway might also be linked to segregated whisker control.

Though research on the vibrissal motor system is still at an early stage, it is clear that analysis of the very fast and sophisticated whisker movements provides rich insight into the cellular mechanisms of movement control.

Acknowledgements This work was supported by the Erasmus MC, a VIDI-grant of the Netherlands Research Organization and a HSFPgrant to $\mathrm{MB}$.

\section{References}

1. Vincent SB (1912) The function of vibrissae in the behavior of the white rat. Behav Monogr 1:7-81

2. Gustafson JW, Felbain-Keramidas SL (1977) Behavioral and neural approaches to the function of the mystacial vibrissae. Psychol Bull 84:477-488

3. Brecht M, Preilowski B, Merzenich MM (1997) Functional architecture of the mystacial vibrissae. Behav Brain Res 84:81-97
4. Jones EG, Diamond IT (1995) The barrel cortex of rodents. Plenum, New York

5. Petersen CC (2003) The barrel cortex-integrating molecular, cellular and systems physiology. Pflugers Arch 447:126-134

6. Kleinfeld D, Berg RW, O’Connor SM (1999) Anatomical loops and their electrical dynamics in relation to whisking by rat. Somatosens Motor Res 16:69-88

7. Ahissar E, Kleinfeld D (2003) Closed-loop neuronal computations: focus on vibrissa somatosensation in rat. Cereb Cortex 13:53-62

8. Welker WI (1964) Analysis of sniffing of the albino rat. Behaviour 22:223-244

9. Wineski LE (1983) Movements of the cranial vibrissae in the golden hamster (Mesocritus auratus). J Zool Lond 200:261-280

10. Lee KJ, Woolsey TA (1975) A proportional relationship between peripheral innervation density and cortical neuron number in the somatosensory system of the mouse. Brain Res 99:349-353

11. Carvell GE, Simons DJ (1990) Biometric analyses of vibrissal tactile discrimination in the rat. J Neurosci 10:2638-2648

12. Jin T, Witzemann V, Brecht M (2004) Fiber types of the intrinsic whisker muscle and whisking behavior. J Neurosci 24: 3386-3393

13. Berg RW, Kleinfeld D (2003) Rhythmic whisking by rat: retraction as well as protraction of the vibrissae is under active muscular control. J Neurophysiol 89:104-117

14. Bermejo R, Harvey M, Gao P, Zeigler HP (1996) Conditioned whisking in the rat. Somatosens Motor Res 13:225-233

15. Bermejo R, Vyas A, Zeigler HP (2002) Topography of rodent whisking-I. Two-dimensional monitoring of whisker movements. Somatosens Motor Res 19:341-346

16. Sachdev RNS, Sato T, Ebner FF (2002) Divergent movements of adjacent whiskers. J Neurophysiol 87:1440-1448

17. Sachdev RN, Berg RW, Champney G, Kleinfeld D, Ebner FF (2003) Unilateral vibrissa contact: changes in amplitude but not timing of rhythmic whisking. Somatosens Motor Res 20:163-169

18. Lerma J, Garcia-Austt E (1985) Hippocampal theta rhythm during paradoxical sleep. Effects of afferent stimuli and phase relationships with phasic events. Electroencephalogr Clin Neurophysiol 60:46-54

19. Timo-Iaria C, Yamashita R, Hoshino K, Souza-Melo A (1990) Rostrum movements in desynchronized sleep as a prevalent manifestation of dreaming activity in Wistar rats. Braz J Med Biol Res 23:617-620

20. Brecht M, Schneider M, Sakmann B, Margrie TW (2004) Whisker movements evoked by stimulation of single pyramidal cells in rat motor cortex. Nature 427:704-710

21. Krupa DJ, Matell MS, Brisben AJ, Oliveira LM, Nicolelis MA (2001) Behavioral properties of the trigeminal somatosensory system in rats performing whisker-dependent tactile discriminations. J Neurosci 21:5752-5763

22. Carvell GE, Simons DJ, Lichtenstein SH, Byrant P (1991) Electromyographic activity of mystacial pad musculature during whisking behavior in the rat. Somatosens Motor Res $8: 159-164$

23. Bermejo R, Houben D, Zeigler HP (1998) Optoelectronic monitoring of individual whisker movements in rats. J Neurosci Methods 83:89-96

24. Knutsen PM, Derdikman D, Ahissar E (2005) Tracking whisker and head movements in unrestrained behaving rodents. J Neurophysiol 93:2294-2301

25. Dörfl J (1982) The musculature of the mystacial vibrissae of the white mouse. J Anat 135:147-154

26. Wineski LE (1985) Facial morphology and vibrissal movement in the golden hamster. J Morphol 183:199-217

27. Hughes SM, Chi MM, Lowry OH, Gundersen K (1999) Myogenin induces a shift of enzyme activity from glycolytic to oxidative metabolism in muscles of transgenic mice. J Cell Biol 145:633-642 
28. Bottinelli R, Schiaffino S, Reggiani C (1991) Force-velocity relationship and myosin heavy chain isoform compositions of skinned fibers from rat skeletal muscle. J Physiol (Lond) 437:655-672

29. Schiaffino S, Reggiani C (1996) Molecular diversity of myofibrillar proteins: gene regulation and functional significance. Physiol Rev 76:371-423

30. Friauf E (1986) Morphology of motoneurons in different subdivisions of the rat facial nucleus stained intracellularly with horseradish peroxidase. J Comp Neurol 253:231-241

31. Klein BG, Rhoades RW (1985) Representation of whisker follicle intrinsic musculature in the facial motor nucleus of the rat. J Comp Neurol 232:55-69

32. Zucker E, Welker WI (1969) Coding of somatic sensory input by vibrissae neurons in the rat's trigeminal ganglion. Brain Res $12: 138-156$

33. Nguyen QT, Wessel R, Kleinfeld D (2004) Developmental regulation of active and passive membrane properties in rat vibrissa motoneurones. J Physiol 556:203-219

34. Hattox AM, Li Y, Keller A (2003) Serotonin regulates rhythmic whisking. Neuron 39:343-352

35. Nguyen QT, Kleinfeld D (2005) Positive feedback in a brainstem tactile sensorimotor loop. Neuron 45:447-457

36. Hattox AM, Priest CA, Keller A (2002) Functional circuitry involved in the regulation of whisker movements. J Comp Neurol 442:266-276

37. Gao P, Bermejo R, Zeigler HP (2001) Vibrissa deaffentation and rodent whisking patterns: behavioral evidence for a central pattern generator. J Neurosci 21:5374-5380

38. Lang EJ, Sugihara I, Llinas R (2006) Olivocerebellar modulation of motor cortex ability to generate vibrissal movements in rat. J Physiol 571:101-120

39. Leonard CM (1969) The prefrontal cortex of the rat. I. Cortical projection of the mediodorsal nucleus. II. Efferent connections. Brain Res 12:321-343

40. Wree A, Lutz B, Thole U (1992) Volumes of the cytoarchitectonic areas in the rat cerebral cortex. J Hirnforsch 33:545-548

41. Brecht M, Krauss A, Muhammad S, Sinai-Esfahani L, Bellanca S, Margrie TW (2004) Organization of rat vibrissa motor cortex and adjacent areas according to cytoarchitectonics, microstimulation, and intracellular stimulation of identified cells. J Comp Neurol 479:360-373

42. Woolsey CN (1958) Organization of somatic sensory and motor areas of the cerebral cortex. In: Harlow HF, Woolsy CN (eds) Biological and biochemical bases of behavior. University of Wisconsin Press, Madison, pp 63-81

43. Hall RD, Lindholm EP (1974) Organization of motor and somatosensory neocortex in the albino rat. Brain Res 66:23-38

44. Gioanni Y, Lamarche M (1985) A reappraisal of rat motor cortex organization by intracortical microstimulation. Brain Res 344: 49-61

45. Miyashita E, Keller A, Asanuma H (1994) Input-output organization of the rat vibrissal motor cortex. Exp Brain Res 99: 223-232

46. Neafsey EJ, Bold EL, Haas, G, Hurley-Gius KM, Quirk G, Sievert CF, Terreberry RR (1986) The organization of the rat motor cortex: a microstimulation mapping study. Brain Res 396: $77-96$

47. Huntley GW (1997) Differential effects of abnormal tactile experience on shaping representation patterns in developing and adult motor cortex. J Neurosci 17:9220-9232

48. Haiss F, Schwarz C (2005) Spatial segregation of different modes of movement control in the whisker representation of rat primary motor cortex. J Neurosci 25:1579-1587

49. Donoghue JP, Wise SP (1982) The motor cortex of the rat: cytoarchitecture and microstimulation mapping. J Comp Neurol 212:76-88
50. Neafsey EJ (1990) The complete ratunculus: output organization of layer V of the cerebral cortex. In: Kolb B, Tees RC (eds) The cerebral cortex of the rat. MIT, Cambridge Ma, pp 197-212

51. Asanuma H (1989) The motor cortex. Raven, New York

52. Welker E, Hoogland PV, Van der Loos H (1988) Organization of feedback and feedforward projections of the barrel cortex: a PHAL study in the mouse. Exp Brain Res 73:411-435

53. Hoffer ZS, Hoover JE, Alloway KD (2003) Sensorimotor corticocortical projections from rat barrel cortex have an anisotropic organization that facilitates integration of inputs from whiskers in the same row. J Comp Neurol 466:525-544

54. Alloway KD, Zhang M, Chakrabarti S (2004) Septal columns in rodent barrel cortex: functional circuits for modulating whisking behavior. J Comp Neurol 480:299-309

55. Grinevich V, Brecht M, Osten P (2005) Monosynaptic pathway from rat vibrissa motor cortex to facial motor neurons revealed by lentivirus-based axonal tracing. J Neurosci 25:8250-8258

56. Kuypers HG (1982) A new look at the organization of the motor system. Prog Brain Res 57:381-403

57. Ahrens KF, Kleinfeld D (2004) Current flow in vibrissa motor cortex can phase-lock with exploratory rhythmic whisking in rat. J Neurophysiol 92:1700-1707

58. Berg RW, Kleinfeld D (2003) Vibrissa movement elicited by rhythmic electrical microstimulation to motor cortex in the aroused rat mimics exploratory whisking. J Neurophysiol 90:2950-2963, 2003

59. Kleinfeld D, Sachdev RNS, Merchant LM, Jarvis MR, Ebner FF (2002) Adaptive filtering of vibrissa input in motor cortex of rat. Neuron 34:1021-1034

60. Carvell GE, Miller SA, Simons DJ (1996) The relationship of vibrissal motor cortex unit activity to whisking in the awake rat. Somatosens Motor Res 13:115-127

61. Brecht M (2004) What makes whiskers shake? Focus on "Current flow in vibrissa motor cortex can phase-lock with exploratory rhythmic whisking in rat". J Neurohypsiol 92:1265-1266

62. Semba K, Komisaruk BR (1984) Neural substrates of two different rhythmical vibrissal movements in the rat. Neuroscience 12:761-774

63. Gao P, Hattox AM, Jones LM, Keller A, Zeigler HP (2003) Whisker motor cortex ablation and whisker movement patterns. Somatosens Motor Res 20:191-198

64. Margrie TW, Brecht M, Sakmann B (2002) In vivo, lowresistance, whole-cell recordings from neurons in the anaesthetized and awake mammalian brain. Pflugers Arch 444:491-498

65. Fritsch G, Hitzig E (1870) Über die elektrische Erregbarkeit des Grosshirns. Arch Anat Physiol Wis Med 37:300-332

66. Asanuma H, Sakata H (1967) Functional organization of a cortical efferent system examined with focal depth stimulation in cats. J Neurophysiol 30:35-54

67. Tehovnik ET (1996) Electrical stimulation of neural tissue to evoke behavioral responses. J Neurosci Methods 65:1-17

68. Woody CD, Black-Cleworth P (1973) Differences in excitability of cortical neurons as a function of motor projection in conditioned cats. J Neurophysiol 36:1104-1116

69. Vallbo AB, Olsson KA, Westberg KG, Clark FJ (1984) Microstimulation of single tactile afferents from the human hand. Sensory attributes related to unit type and properties of receptive fields. Brain 107:727-749

70. Vallbo AB (1981) Sensations evoked from the glabrous skin of the human hand by electrical stimulation of unitary mechanosensitive afferents. Brain Res 215:359-363

71. Burke RE (1994) Physiology of motor units. In: Engel AG, Franzini-Armstrong C (eds) Myology, 2nd edn. McGraw-Hill, New York, pp 464-483

72. Paxinos G, Watson C (1998) The rat brain in stereotactic coordinates, 4th edn. Academic, New York 